This is an electronic reprint of the original article. This reprint may differ from the original in pagination and typographic detail.

Author(s): Kronholm, Risto; Kalvas, Taneli; Koivisto, Hannu; Tarvainen, Olli

Title: $\quad$ Spectroscopic method to study low charge state ion and cold electron population in ECRIS plasma

Year: $\quad 2018$

Version:

Please cite the original version:

Kronholm, R., Kalvas, T., Koivisto, H., \& Tarvainen, O. (2018). Spectroscopic method to study low charge state ion and cold electron population in ECRIS plasma. Review of Scientific Instruments, 89(4), Article 043506. https://doi.org/10.1063/1.5023434

All material supplied via JYX is protected by copyright and other intellectual property rights, and duplication or sale of all or part of any of the repository collections is not permitted, except that material may be duplicated by you for your research use or educational purposes in electronic or print form. You must obtain permission for any other use. Electronic or print copies may not be offered, whether for sale or otherwise to anyone who is not an authorised user. 


\section{Spectroscopic method to study low charge state ion and cold electron population in ECRIS plasma}

R. Kronholm, T. Kalvas, H. Koivisto, and O. Tarvainen

Citation: Review of Scientific Instruments 89, 043506 (2018); doi: 10.1063/1.5023434

View online: https://doi.org/10.1063/1.5023434

View Table of Contents: http://aip.scitation.org/toc/rsi/89/4

Published by the American Institute of Physics

\section{Articles you may be interested in}

Broadband microwave emission spectrum associated with kinetic instabilities in minimum-B ECR plasmas

Physics of Plasmas 24, 043515 (2017); 10.1063/1.4981387

Improving the viability and versatility of the $\mathrm{E} \times \mathrm{B}$ probe with an active cooling system

Review of Scientific Instruments 89, 043502 (2018); 10.1063/1.5004469

Calibrated work function mapping by Kelvin probe force microscopy

Review of Scientific Instruments 89, 043702 (2018); 10.1063/1.5007619

Effective ion charge $\left(Z_{\text {eff }}\right)$ measurements and impurity behavior in KSTAR

Review of Scientific Instruments 89, 043504 (2018); 10.1063/1.5004217

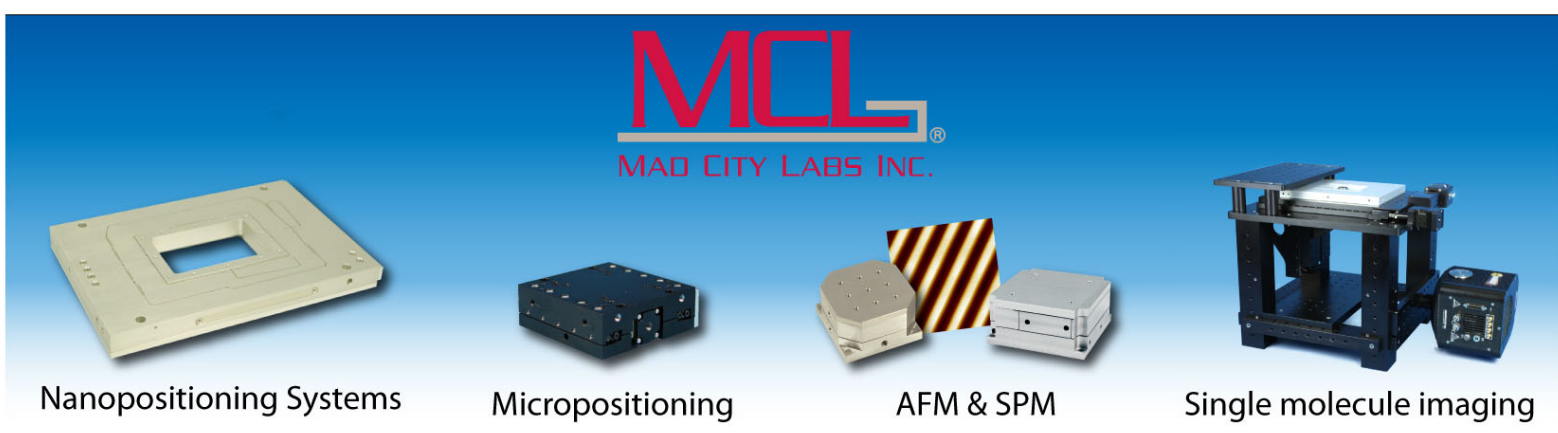




\title{
Spectroscopic method to study low charge state ion and cold electron population in ECRIS plasma
}

\author{
R. Kronholm, ${ }^{\text {a) }}$ T. Kalvas, H. Koivisto, and O. Tarvainen \\ Department of Physics, University of Jyväskylä, Jyväskylä, Finland
}

(Received 24 January 2018; accepted 15 March 2018; published online 9 April 2018)

\begin{abstract}
The results of optical emission spectroscopy experiments probing the cold electron population of a $14 \mathrm{GHz}$ Electron Cyclotron Resonance Ion Source (ECRIS) are reported. The study has been conducted with a high resolution spectrometer and data acquisition setup developed specifically for the diagnostics of weak emission line characteristic to ECRIS plasmas. The optical emission lines of low charge state ions and neutral atoms of neon have been measured and analyzed with the line-ratio method. The aforementioned electron population temperature of the cold electron population $\left(T_{e}<\right.$ $100 \mathrm{eV}$ ) is determined for Maxwell-Boltzmann and Druyvesteyn energy distributions to demonstrate the applicability of the method. The temperature was found to change significantly when the extraction voltage of the ion source is turned on/off. In the case of the Maxwellian distribution, the temperature of the cold electron population is $20 \pm 10 \mathrm{eV}$ when the extraction voltage is off and $40 \pm 10 \mathrm{eV}$ when it is on. The optical emission measurements revealed that the extraction voltage also affects both neutral and ion densities. Based on the rate coefficient analysis with the aforementioned temperatures, switching the extraction voltage off decreases the rate coefficient of neutral to $1+$ ionization to $42 \%$ and $1+$ to $2+$ ionization to $24 \%$ of the original. This suggests that switching the extraction voltage on favors ionization to charge states $\geq 2+$ and, thus, the charge state distributions of ECRIS plasmas are probably different with the extraction voltage on/off. It is therefore concluded that diagnostics results of ECRIS plasmas obtained without the extraction voltage are not depicting the plasma conditions in normal ECRIS operation. Published by AIP Publishing. https://doi.org/10.1063/1.5023434
\end{abstract}

\section{INTRODUCTION}

At the JYFL (University of Jyväskylä, Department of Physics) accelerator laboratory, electron cyclotron resonance ion sources ${ }^{1}$ (ECRISs) are used for the production of highly charged heavy ion beams for an active nuclear physics program. In ECR ion source plasma, free electrons are heated as a result of energy transfer from the microwave electric field to the electrons in relativistic electron cyclotron resonance at the magnetic field isosurface where $\omega_{c e}=\omega_{R F}$, i.e., when the electron gyrofrequency equals the frequency of the injected microwave radiation. The resulting electron distribution can be divided into cold, warm, and hot populations with different energies (10-100 eV, 0.1-10 keV, and 10-100 keV). ${ }^{2,3}$ Cold electrons, which are believed to have energies on the order of some tens of eVs, are produced in ionization reactions and contribute significantly to the ionization of neutral atoms and low charge state ions. Warm and hot electrons have gained energy from the microwaves in resonance. Warm electrons with energies of some keVs are responsible for high charge state ion production while hot (run-away) electrons affect the plasma confinement, e.g., by presumably creating a potential dip $^{4}$ in the plasma core.

The main purpose of ECRIS is most often high charge state ion production. While the focus of the ion source development and diagnostics is usually in the high charge state ions and electron populations contributing to their ionization and confinement, also cold electrons, neutral atoms, and low charge

a)Electronic mail: risto.j.kronholm@jyu.fi state ions play a significant role in ECRIS plasma dynamics. The equilibrium of the charge state distribution depends on the volumetric rates of ionization and charge exchange reactions as well as the ion confinement time. ${ }^{5}$ The volumetric rate of the charge exchange reactions is strongly affected by the neutral gas density as the cross sections for charge exchange are often several orders of magnitude higher in comparison to ionization cross sections of highly charged ions. ${ }^{6,7}$ It has also been shown that the low charge state ions play an important role in the physics of the gas mixing technique used to increase the high charge state ion production in ECRIS plasmas. ${ }^{8}$

Studying low charge state ions, neutral atoms, and cold electrons in ECRIS plasmas is challenging. The main diagnostic method for low charge state ions is beam extraction which is sometimes hampered by insufficient ion optics for the ions with high M/Q-values. Meanwhile, the only widely available diagnostic method to study the energies of the cold electrons is the invasive Langmuir-probe technique, ${ }^{9}$ which inherently affects the plasma properties, e.g., the charge state distribution. Due to the spontaneous emissions of electronic transitions between the excited states of ions and neutrals, electromagnetic radiation is emitted which opens a possibility to study ECRIS plasmas with optical emission spectroscopy (OES), which is a non-invasive technique. Further benefit of OES is that the signals are hardly affected by the rf-fields, static magnetic fields, or electrostatic potentials present in the ECRIS environment. The purpose of this work is to introduce a method to study relative densities of low charge state ions and neutral atoms and probe the temperature of the cold electron population in the range of 10-100 eV with high-resolution OES. The OES 
technique has been used for high frequency ECRIS plasma diagnostics in the past by several authors. ${ }^{10-12}$ For example, Bibinov et al. have introduced a spectroscopic method for determining the energy distribution of $<10 \mathrm{eV}$ electrons, i.e., at energies below the plasma potential in ECRIS plasmas. ${ }^{13}$ The method is based on identifying vibrational structures in the emission spectra of nitrogen and the analysis of the relevant excitation rates.

In order to demonstrate the applicability of the diagnostics method described hereafter, the technique was applied to study the effect of the ion source extraction voltage $(10 \mathrm{kV})$ on the temperature of the low energy electron population and the ratio of $1+$ and neutral densities in neon plasmas of a $14 \mathrm{GHz}$ ECRIS. The effect of the high voltage is often not taken into account when studying ECRIS plasmas. It has been observed indirectly that switching the high voltage on/off does not affect the bremsstrahlung spectrum created by the hot electron population at energies $>100 \mathrm{keV} .{ }^{14}$ On the other hand, the high voltage has been shown to have a strong effect on the magnetic field threshold of kinetic instabilities characteristic to non-equilibrium ECRIS plasmas. ${ }^{15}$ This suggests that the high voltage affects the ratio between the densities of cold and hot electron populations. It is demonstrated here that switching the high voltage on/off affects both the cold electron and low charge state ion populations and, thus, cannot be ignored when performing plasma diagnostics with ECRIS.

\section{THEORETICAL BACKGROUND}

Free electrons that have gained energy through the ECR can transfer energy to electrons bound to the electric potential of an atom or an ion through inelastic electron impact collisions. As a result of such collisions, the bound electron can be excited to a higher energy state or escape from the potential well created by the positively charged atomic nucleus. These processes are called electron impact excitation and electron impact ionization, respectively.

The underlying principle of optical emission spectroscopy applied to ECRIS plasmas is that the electron occupies the excited states for a very short time and then returns to a lower energy state. The energy of the photons emitted in electronic transitions from the upper energy level $j$ to the lower energy level $k$ corresponds to the difference of the energy states $E_{j}$ and $E_{k}$. The wavelength of the emitted photon is given by

$$
\lambda=h c /\left(E_{j}-E_{k}\right),
$$

where $h$ is the Planck constant and $c$ is the speed of light. The described photon emission process accounts for the spontaneous emission of electromagnetic radiation of ECRIS plasmas in a wide range of wavelengths spanning from x-rays to infrared. In the following, we have limited our research to cover an extended visible light wavelength range from 300 to $800 \mathrm{~nm}$. The radiation in this range originates from atomic and molecular electronic transitions between the outer shells of the atoms and compounds.

The intensity of the emission, $I_{j k}$, in units of photons/ $\left(\mathrm{m}^{3} \mathrm{~s}\right)$, depends on the population density $n_{j}$ of the electronic state $j$ and the constant transition probability $A_{j k}$ as

$$
I_{j k}=n_{j} A_{j k} .
$$

The intensity of the emitted radiation (number of photons) is directly connected to the population rate of the excited state $j$ under the assumption of the so-called corona equilibrium. ${ }^{16}$ The dominant population mechanism of the electronic state $j$ in a plasma is excitation from lower lying energy states by direct electron impact collision or by photon absorption. The state $j$ can also be populated by radiative or non-radiative transitions from higher energy states, recombination reactions between free electrons and ions, electron impact ionization of lower charge state ions, or charge exchange reactions mainly between ions and neutrals.

The existence of the corona equilibrium in ECRIS plasmas is supported by the low (on the order of $10^{12} \mathrm{~cm}^{-3}$ as a maximum) electron density and high average electron energy (discussed earlier). ${ }^{17}$ On the other hand, the radiation density can be considered negligible, which assures that the volumetric rate of electron impact excitations greatly exceeds the rate of excitations by photon absorption. In ECRIS plasmas, the dominant particle collision process is ion-ion collisions, ${ }^{18}$ with frequencies on the order of $10^{6}-10^{7} \mathrm{~Hz}$ corresponding to 0.1-1 $\mu$ s mean time between ion-ion collisions. Electron-ion and electron-neutral collisions occur at a lower frequency and, therefore, it is concluded that the typical nanosecond lifetimes of the spontaneously emitting states are at least 2-3 orders of magnitude lower than the time between particle collisions. Owing to the high electron energies and small cross sections, the electron recombination rate between free electrons and $1+$ ions is deemed small in comparison to the electron impact excitation rate. ${ }^{19}$ Excitation by photon re-absorption can be neglected due to low neutral and 1+ densities, i.e., ECRIS plasmas can be considered optically thin. Yet another reaction, in which the ion ends up to an excited state, is charge exchange between a highly charged ion and neutral atom. It has been observed that the resulting $1+$ target ions generated in this process are predominantly on their ground state, unlike the projectile ions. ${ }^{20}$ On this basis, the effect of the charge exchange is ignored for $1+$ neon ions. Finally, the contribution from radiative decays from higher energy levels can be measured and, therefore, taken into account when estimating the population rate of an electronic state $j$. Altogether, electron impact excitation from ground or metastable states can be assumed to be the main process populating the excited electronic states in ECRIS plasmas.

When dealing with collisions between free electrons and atoms or ions, it is convenient to introduce the quantity of collision strength $\Omega$ to describe the probabilities of optical transitions. The collision strength is related to the collision cross section $\sigma$ by

$$
\sigma_{i j}=\frac{h^{2}}{8 \pi m_{e} E} \frac{\Omega_{i j}(E)}{g_{i}},
$$

where $m_{e}$ is the electron mass, $E$ is the kinetic energy of the colliding electron, and $g_{i}$ is the statistical weight of the initial electronic state. Electron impact reaction rates in units of $\mathrm{s}^{-1}$ $\mathrm{m}^{-3}$ are described as

$$
\begin{aligned}
R_{i j} & =n_{e} n_{q} \int \sigma_{i j} v_{e} f_{e}(v) d v \\
& =n_{e} n_{q}\left\langle\sigma_{i j} v\right\rangle=n_{e} n_{q} \gamma_{i j}
\end{aligned}
$$


where $n_{e}$ is the electron density, $n_{q}$ is the target (neutral or ion) density, $v_{e}$ is the velocity of projectile electrons, $f_{e}$ is the electron velocity distribution function (EVDF), and $\gamma_{i j}$ is the rate coefficient for the reaction. The EVDF, usually presented as the electron energy distribution function EEDF, in ECRIS plasmas is not well-known but is often approximated with several overlapping Maxwell-Boltzmann distributions weighted by the assumed electron densities of each population. However, we emphasize that the principles of the diagnostics suitable for probing the cold electron population presented in Secs. II and IV are not critically sensitive to the EVDF. Two different distributions, Maxwell-Boltzmann and Druyvesteyn, are used to illustrate this point. They act as mere examples simplifying the mathematics and allowing us to describe the EVDF with a single characteristic figure, the electron temperature, $T_{e}$. The Maxwell-Boltzmann distribution represents the velocity distribution for a gas in equilibrium, while the Druyvesteyn distribution corresponds to a non-equilibrium gas under certain assumptions. ${ }^{21}$ The Druyvesteyn distribution has been derived for discharge plasmas, where the electron temperature is higher than the ion and neutral temperatures and the prevailing interaction between the studied electron population and heavier particles is dominated by collisions with neutrals, which can be assumed to hold relatively well for the cold electron population of an ECRIS. The isotropic Maxwell-Boltzmann velocity distribution is written as

$$
f_{M-B}(v)=4 \pi\left(\frac{m_{e}}{2 \pi k T_{e}}\right)^{3 / 2} v^{2} \exp \left(\frac{-m_{e} v^{2}}{2 k T_{e}}\right)
$$

and the Druyvesteyn distribution as

$f_{D}(v)=\frac{1.04 \pi^{1 / 2}}{2}\left(\frac{m_{e}}{2 \pi k T_{e}}\right)^{3 / 2} v^{2} \exp \left(-0.55\left(\frac{m_{e} v^{2}}{2 k T_{e}}\right)^{2}\right)$.

The comparison between the Maxwell-Boltzmann and Druyvesteyn distributions is presented in Fig. 1. The corresponding rate coefficients are expressed as

$$
\gamma_{i j, M B}\left(T_{e}\right)=4 \pi\left(\frac{m_{e}}{2 \pi k T_{e}}\right)^{3 / 2} \int v^{3} \sigma_{i j}(v) \exp \left(\frac{-m_{e} v^{2}}{2 k T_{e}}\right) d v
$$

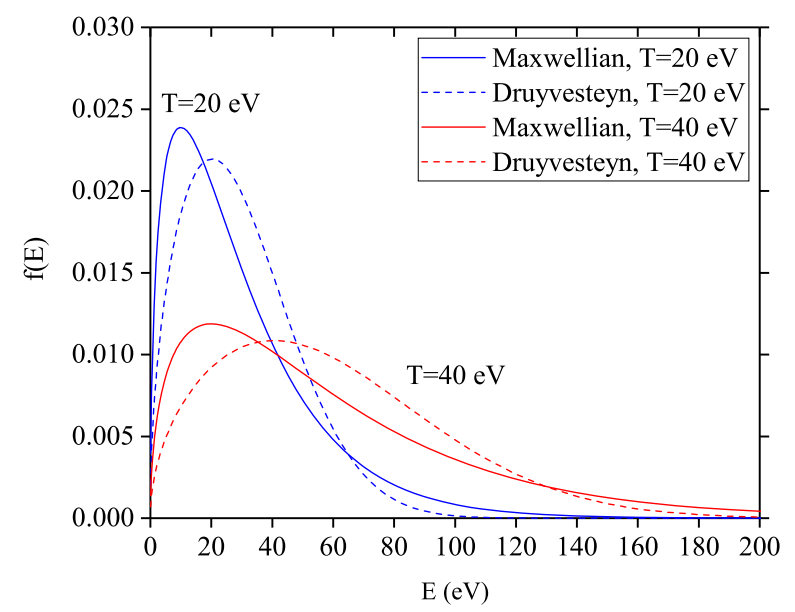

FIG. 1. Comparison between Maxwell-Boltzmann and Druyvesteyn distributions. The distributions are normalized to constant particle density. and

$$
\begin{aligned}
\gamma_{i j, D}\left(T_{e}\right)= & \frac{1.04 \pi^{1 / 2}}{2}\left(\frac{m_{e}}{2 \pi k T_{e}}\right)^{3 / 2} \int v^{3} \sigma_{i j}(v) \\
& \times \exp \left(-0.55\left(\frac{m_{e} v^{2}}{2 k T_{e}}\right)^{2}\right) d v .
\end{aligned}
$$

In the corona equilibrium, the balance between the excitation and de-excitation (emission) rates is

$$
n_{e} n_{q} \gamma_{i j}=n_{j} \sum_{k} A_{j k}=\sum_{k} I_{j k} .
$$

The emission intensities involving transitions from state $j$ to state $k$ are affected by the branching ratio describing the decay probabilities to competing lower electronic states. If, for example, the upper state $j$ decays radiatively to lower states $k=1$, $2, \ldots, n$ with transition probabilities of $A_{j 1}, A_{j 2}, \ldots, A_{j n}$, the total radiative lifetime can be obtained from

$$
\frac{1}{\tau}=\sum_{k=1}^{n} A_{j k}
$$

The branching ratio $B R_{j k}$ of the transition to state $k$ is defined as

$$
B R_{j k}=\frac{A_{j k}}{\sum_{k=1}^{n} A_{j k}} .
$$

The branching ratio can be determined by measuring the intensities of all spectral lines corresponding to the de-excitation of the upper state $j$. Finally, the emission intensity of a specific transition from the upper level $j$ to level $k$ can be connected to the total electron impact excitation rate to state $j$ using the branching ratio, i.e.,

$$
I_{j k}=n_{e} n_{q} \gamma_{i j} B R_{j k} .
$$

The temperature of the electrons responsible for the excitations to the upper level $j$ can be studied by measuring the intensities of the emission lines and comparing the excitation rates from the same lower energy state to different higher energy states $j=a$ and $j=b$ having excitation cross sections of different functional shapes (electron energy dependencies). The observed emission intensities can be connected to the rate coefficients, and thus to electron temperatures, through

$$
\frac{I_{a k}}{I_{b k}} \approx \frac{n_{e} n_{q} \gamma_{a} B R_{a k}}{n_{e} n_{q} \gamma_{b} B R_{b k}}=\frac{\gamma_{a} B R_{a k}}{\gamma_{b} B R_{b k}} .
$$

The reliability of the analysis can be increased by comparing the ratios of multiple emission lines. Furthermore, if the transitions $a$ and $b$ originate from different charge states $q 1$ and $q 2$ of the ions and the electron temperature can be deduced from Eq. (13), the ratio of the densities of charge states $q 1$ and $q 2$ can be obtained from

$$
\frac{I_{a k}}{I_{b k}} \approx \frac{n_{e} n_{q 1} \gamma_{a} B R_{a k}}{n_{e} n_{q 2} \gamma_{b} B R_{b k}}=\frac{n_{q 1}}{n_{q 2}} \frac{\gamma_{a} B R_{a k}}{\gamma_{b} B R_{b k}} .
$$

The line-ratio method is commonly used in low temperature plasmas. ${ }^{16}$ In this work, the described diagnostics method was applied for a neon plasma of a high-frequency ECRIS to study the effect of the source high voltage on the temperature of the low energy electron population and the ratio of 
TABLE I. Observed transitions from level $j$ to level $k$.

\begin{tabular}{llccccc}
\hline \hline Transition & Ion & Wavelength $(\mathrm{nm})$ & Levels $(\mathrm{k}-\mathrm{j})$ & $E_{k}(\mathrm{eV})$ & $E_{j}(\mathrm{eV})$ & $A_{j k}\left(10^{7} \mathrm{~s}^{-1}\right)$ \\
\hline 1 & $\mathrm{Ne}$ & 534.109 & $2 \mathrm{p}^{5}\left({ }^{2} \mathrm{P}_{3 / 2}^{\circ}\right) 3 \mathrm{p}^{2}[1 / 2]_{1}-2 \mathrm{p}^{5}\left({ }^{2} \mathrm{P}_{3 / 2}^{\circ}\right) 4 \mathrm{~d}^{2}[1 / 2]_{1}^{\circ}$ & 18.38 & 20.70 \\
2 & $\mathrm{Ne}$ & 607.434 & $2 \mathrm{p}^{5}\left({ }^{2} \mathrm{P}_{3 / 2}^{\circ}\right) 3 \mathrm{~s}^{2}[3 / 2]_{1}^{\circ}-2 \mathrm{p}^{5}\left({ }^{2} \mathrm{P}_{3 / 2}\right) 3 \mathrm{p}^{2}[1 / 2]_{0}$ & 16.67 & 18.71 \\
3 & $\mathrm{Ne}$ & 609.616 & $2 \mathrm{p}^{5}\left({ }^{2} \mathrm{P}_{3 / 2}^{\circ}\right) 3 \mathrm{~s}^{2}[3 / 2]_{1}-2 \mathrm{p}^{5}\left({ }^{2} \mathrm{P}_{1 / 2}\right) 3 \mathrm{p}^{2}[3 / 2]_{2}$ & 16.67 & 18.70 \\
4 & $\mathrm{Ne}^{1+}$ & 332.374 & $2 \mathrm{p}^{4}\left({ }^{3} \mathrm{P}\right) 3 \mathrm{~s}^{2} \mathrm{P}_{3 / 2}-2 \mathrm{p}^{4}\left({ }^{3} \mathrm{P}\right) 3 \mathrm{p}^{2} \mathrm{P}_{3 / 2}^{\circ}$ & 27.78 & 31.51 & 1.8 \\
5 & $\mathrm{Ne}^{1+}$ & 348.072 & $2 \mathrm{p}^{4}\left({ }^{1} \mathrm{~S}\right) 3 \mathrm{~s}^{2} \mathrm{~S}_{1 / 2}-2 \mathrm{p}^{4}\left({ }^{1} \mathrm{~S}\right) 3 \mathrm{p}^{2} \mathrm{P}_{3 / 2}^{\circ}$ & 34.30 & 37.87 \\
6 & $\mathrm{Ne}^{1+}$ & 375.378 & $2 \mathrm{p}^{4}\left({ }^{3} \mathrm{P}\right) 3 \mathrm{p}^{2} \mathrm{P}_{3 / 2}^{\circ}-2 \mathrm{p}^{4}\left({ }^{3} \mathrm{P}\right) 3 \mathrm{~d}^{4} \mathrm{~F}_{5 / 2}^{\circ}$ & 31.51 & 34.81 & 16.0 \\
\hline \hline
\end{tabular}

$1+$ ion to neutral density. The advantage of neon is that it is a noble gas and, thus, does not form molecules or accumulate on the plasma chamber walls. Neon has many suitable excitations with cross sections of different functional shapes. Furthermore, the cross section and branching ratio data are readily available (see below). Six transitions, with sufficient emission intensity of the transition and connected transitions in the ECRIS plasma, were chosen for this study, three of them originating from neutral neon and three from $1+$ ions. These transitions are listed in Table I.

The electron impact excitation cross sections from the ground states of $\mathrm{Ne}^{0+}$ and $\mathrm{Ne}^{1+}$ to the upper states corresponding to the observed emission lines have been calculated from Eq. (3) using the collision strengths obtained from the distorted wave approximation calculation used in the LANL ACE-code described in Refs. 22-24. It has been reported in Ref. 24 that the discrepancy, experimental collision strengths, and those calculated with the distorted wave approximation are less than $10 \%$ in the worst case. The rate coefficients for observed $\mathrm{Ne}^{0+}$ and $\mathrm{Ne}^{1+}$ transitions have been calculated for both Maxwell-Boltzmann and Druyvesteyn distributions. The cross sections and rate coefficients for the Maxwell-Boltzmann distribution are presented in Fig. 2 for electron temperatures of 0-100 eV.
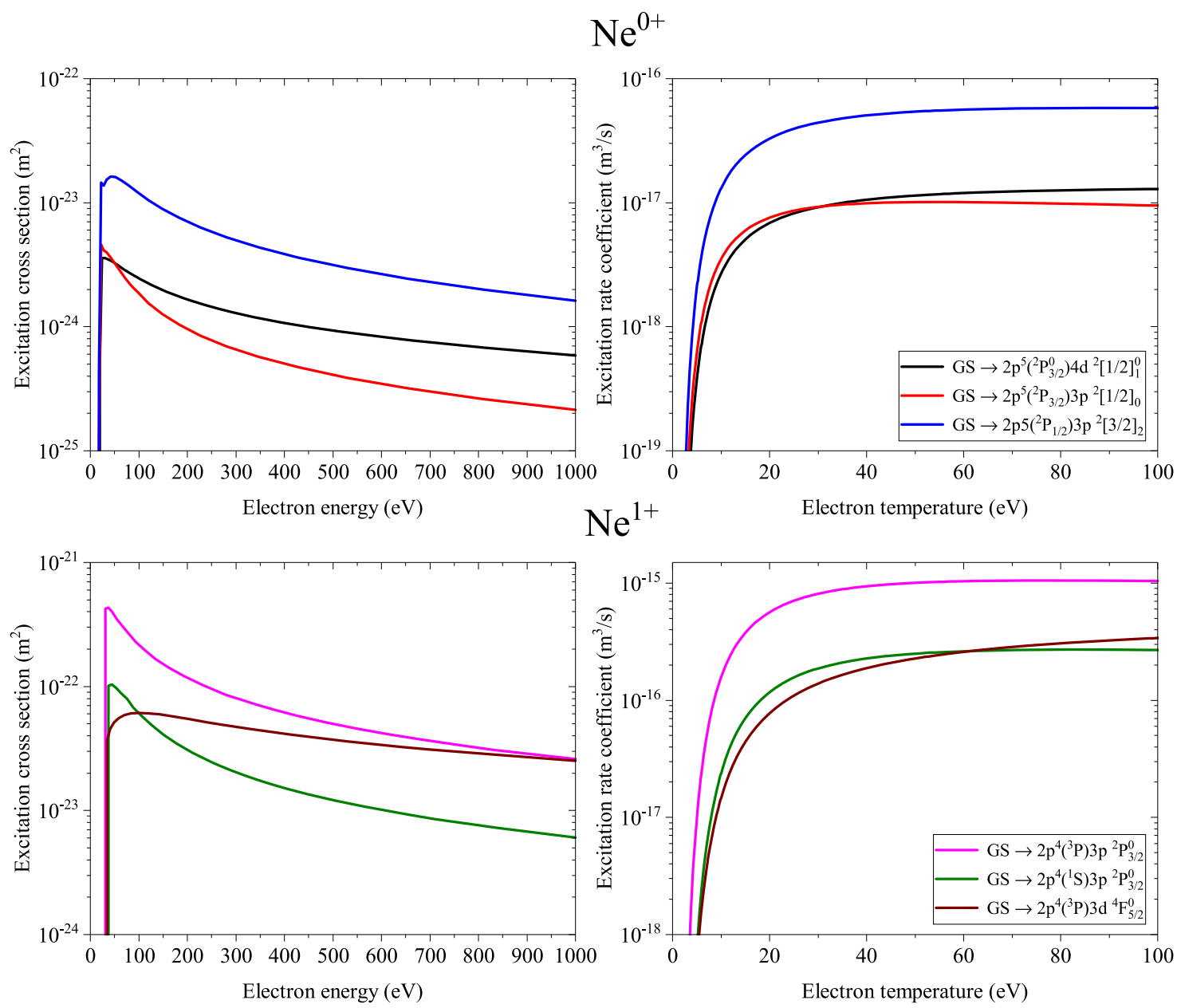

FIG. 2. Calculated electron impact excitation cross sections and Maxwell averaged rate coefficients from the ground state (GS) to the upper states of the transitions listed in Table I. 


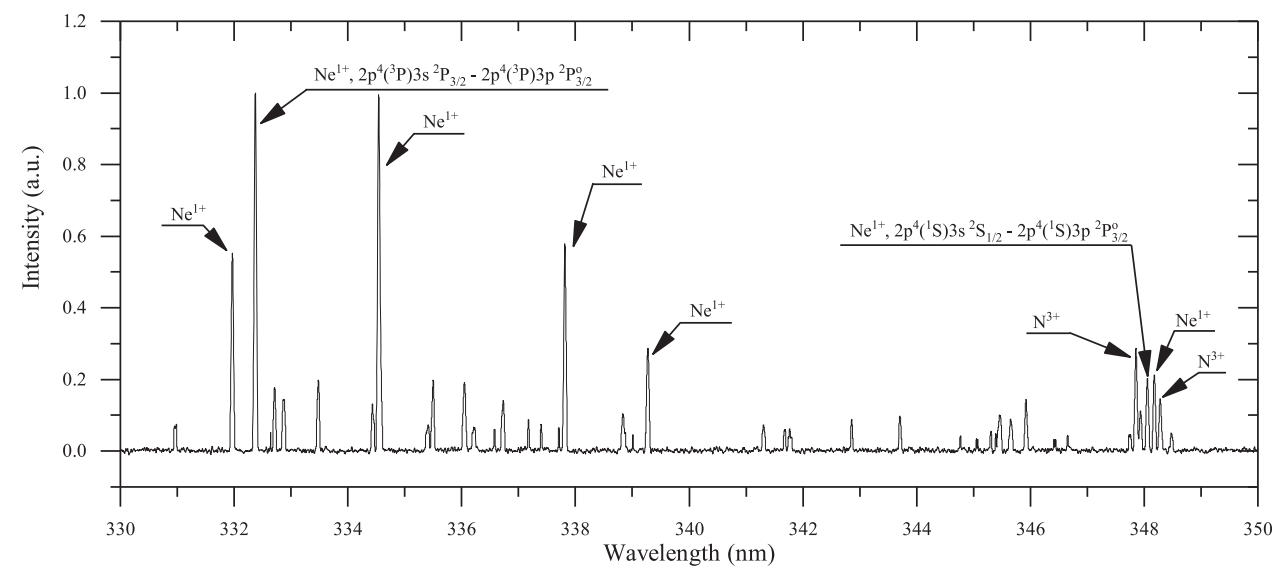

FIG. 3. An example of the optical emission spectrum of a neon plasma in the range of $330 \mathrm{~nm}-350 \mathrm{~nm}$. The charge states corresponding to the most intense emission lines are identified, and the complete spectroscopic notation is presented for the transitions used in this study (see transitions 4 and 5 in Table I).

\section{EXPERIMENTAL SETUP}

The experimental optical emission data were taken with the JYFL $14 \mathrm{GHz}$ ECRIS. The magnetic field of the ECRIS is a superposition of axial and sextupole magnetic fields generated by solenoid coils and a permanent magnet array, thus enabling the production of highly charged ions in minimum-B configuration. The plasma is sustained by microwave radiation at the frequency of $14.1 \mathrm{GHz}$. More detailed information about the JYFL 14 GHz ECRIS can be found in Ref. 1.

In the case of heavy ion plasmas, the number of emission lines in the visible light range is high. As described in Sec. II, a sufficient number of emission lines with known excitation cross sections and threshold energies have to be identified and used for estimating the electron temperature. In order to separate the emission lines listed in Table I from adjacent lines, the instrumental full width at half maximum (FWHM) of the monochromator must be less than $100 \mathrm{pm}$, which is satisfied by the $31 \mathrm{pm} \mathrm{FWHM}$ of the setup described hereafter (see an example spectrum in Fig. 3). In addition, the emission intensities of the optical transitions of interest, especially those of the high charge states can be very low, thus requiring a high optical throughput and high signal-to-noise-ratio (SNR) data acquisition system. The OES setup used for the characterization of the ECRIS plasma consists of three main parts presented schematically in Fig. 4: The optical interface between the ion

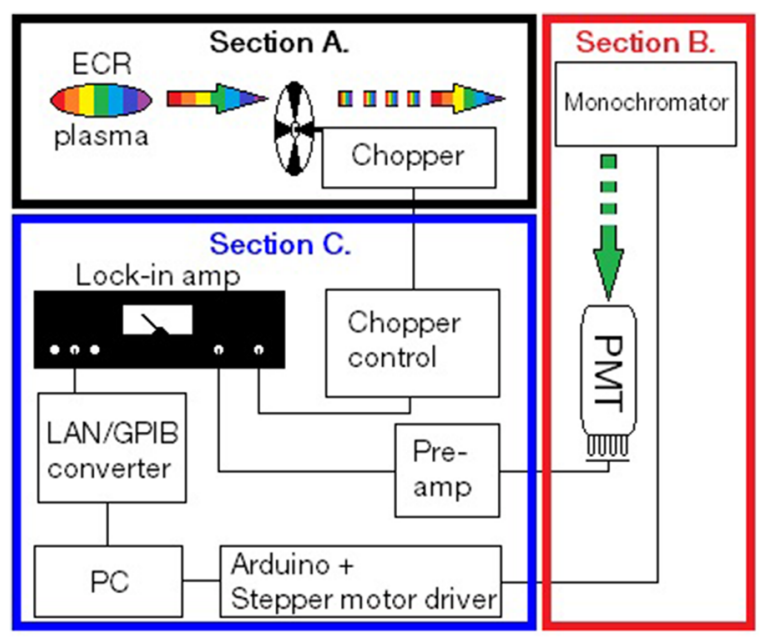

FIG. 4. A schematic diagram of the measurement setup. source and the spectrometer (Sec. III A), the spectrometer, i.e., monochromator coupled with a photomultiplier detector (Sec. III B), and the data acquisition and control system (Sec. III C).

\section{A. The optical interface between the ECRIS plasma and the spectrometer}

The mechanical structure of the JYFL $14 \mathrm{GHz}$ ECRIS offers three optical access points to view the plasma (see Fig. 5): (1) the off-axis oven port in the injection iron plug, (2) the extraction aperture, and (3) the radial pumping ports. Each of the optical access points has a different line-of-sight to the plasma. As the photons are emitted to a full solid angle by each differential plasma volume element, only part of the total emission can be detected. The radial view was chosen as it offers a projection through the plasma core surrounded by the resonance zone.

The vacuum interface indicated in Fig. 5 is a flat $3 \mathrm{~mm}$ thick UV fused silica window with a transmission of

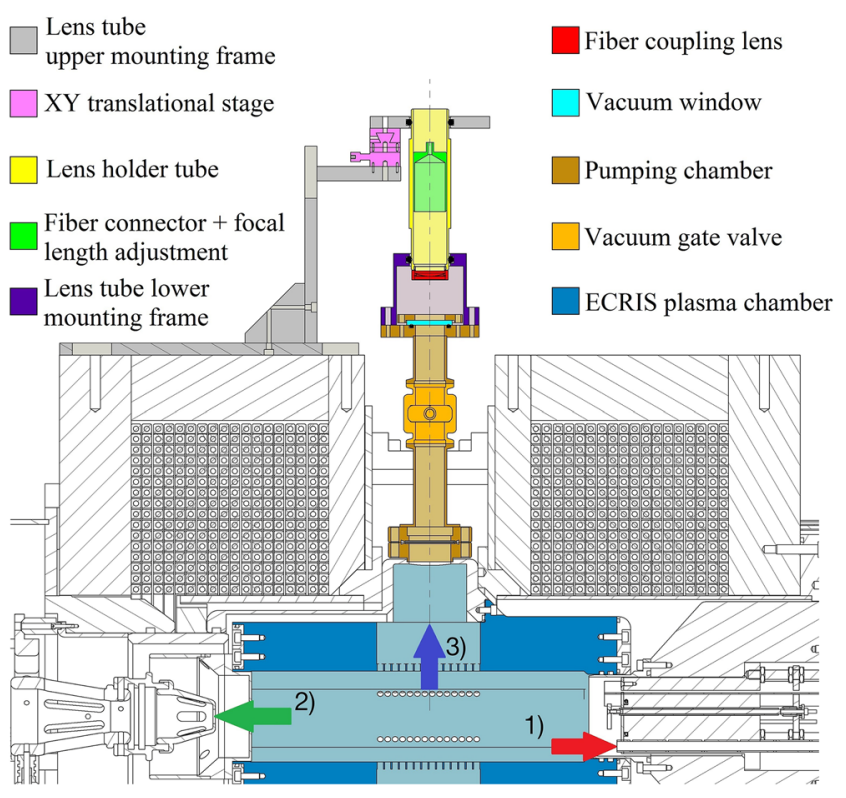

FIG. 5. The cross section view of the optical output system attached to the JYFL 14 GHz ECRIS plasma chamber. Red, green, and blue arrows indicate possible ports for OES studies. 
$93.4 \pm 0.5 \%$ in the wavelength range of $300-800 \mathrm{~nm}$. The vacuum window is followed by a focusing lens, which is used for efficient coupling of the light emanating through a single pumping hole (diameter $6 \mathrm{~mm}$ ) to the optical fiber. The lens is an uncoated UV fused silica Bi-convex singlet lens with an effective diameter of $24 \mathrm{~mm}$ and focal length of $75 \mathrm{~mm}$. The transmission of the lens is $93.4 \pm 0.5 \%$ in the wavelength range of $300-800 \mathrm{~nm}$. The fiber is a $3 \mathrm{~m}$ long, high voltage (HV) compatible, Thorlabs M93L multimode optical fiber having a core diameter of $1.5 \mathrm{~mm}$, numerical aperture of 0.39 , and maximum attenuation of $700 \mathrm{~dB} \mathrm{~km}^{-1}$ in the wavelength range of 300-1200 nm. The focal length adjustment between the lens and the fiber is implemented to the fiber holder indicated in Fig. 5. The fiber holder moves inside the lens holder tube and can be fixed to the optimal position with a locking screw.

The JYFL $14 \mathrm{GHz}$ ECRIS is typically operated at high voltages ranging from $8 \mathrm{kV}$ to $14 \mathrm{kV}$. The optical fiber serves as an insulator between the ECRIS and the monochromator at the ground potential. The light emanating from the fiber is transported into an optical chopper needed for the phase sensitive lock-in detection setup. The chopper system is MC2000BEC with a MC1F60 blade from Thorlabs. The chopper pulses the light in time with a specific chopping frequency set to $f_{c}=5683 \mathrm{~Hz}$ in the experiments described hereafter. The chopping frequency was determined by measuring the noise spectrum from the photomultiplier tube (PMT) pre-amplifier and selecting the frequency with the minimum intensity to maximize the SNR. The light passing through the chopper is coupled to a custom-made multimode fiber consisting of 100 pieces of $100 \mu \mathrm{m}$ core fibers bundled together to form a linear column to mimic the shape of the monochromator input slit, thus optimizing the system throughput and resolution.

\section{B. Spectrometer}

The wavelength separation of the incident light is realized by a $1 \mathrm{~m}$ focal length Fastie-Ebert type monochromator. The components of the setup are the input and output slits, a reflective diffraction grating, and a spherical mirror. The linear end of the fiber bundle acts as a $100 \mu \mathrm{m} \times 10 \mathrm{~mm}$ input slit. The spherical mirror is used to collimate and focus the light from the input slit to the diffraction grating. The diffraction grating is a Spectrogon 2200 grooves $/ \mathrm{mm}$ holographic grating optimized for $450 \mathrm{~nm}$ wavelength. The absolute efficiency of the grating is $40 \%-70 \%$ in the range of $300-800 \mathrm{~nm}$. The rotation angle of the dispersive grating is used for selecting a narrow range of wavelengths reflected back to the spherical mirror and finally focused through the $100 \mu \mathrm{m} \times 10 \mathrm{~mm}$ exit slit. The light passing through the exit slit is coupled to the ETEnterprises 9816B photomultiplier tube (PMT) detector. The nominal sensitivity of the detector is $5000 \mathrm{~A} 1 / \mathrm{m}$ at a nominal voltage of $2084 \mathrm{~V}$ in the spectral range of $300-800 \mathrm{~nm}$. The photomultiplier tube is cooled with a Peltier element to decrease thermal noise and shielded against magnetic fringe fields. The whole spectrometer setup including optics and data acquisition is built on a vibration damped stand.

The spectral gain of the complete measurement system including the optical coupling (window, lens, fiber), monochromator, and PMT at the voltage of $1800 \mathrm{~V}$ has been measured using Ocean Optics HL-3 PLUS-CAL-EXT halogen calibration lamp in the wavelength range of $350-800 \mathrm{~nm}$. The calibration is extended to $330-350 \mathrm{~nm}$ by measuring emission intensities of transition pairs originating from the same upper level and decaying to different lower levels with known branching ratios. The pairs were selected so that one of the transitions is in the range of 330-350 nm and the other within the range covered by the calibration lamp spectrum. The normalized spectral gain measured with the calibration lamp is presented in Fig. 6 as a solid black line, and the calculated results are presented as red dots. All the measured intensities presented in this paper including the spectrum in Fig. 3 have been normalized by using the given spectral gain.

The instrumental broadening of the monochromator depends on the widths of the input and exit slits, grating groove density, and focal length. The instrumental FWHM of the emission lines measured with the described setup was obtained by measuring the $632.991 \mathrm{~nm}$ emission line from a stabilized helium-neon laser (Thorlabs HRS015B). The measurement was carried out with the laser because its FWHM is well known $(1.8 \mathrm{pm})$ and the intensity stability is specified $(<0.3 \%)$. To obtain sufficient statistics, the given emission line was scanned 100 times yielding an average and standard deviation of $31 \pm 1 \mathrm{pm}$ for the instrumental FWHM of the spectrometer at $632.991 \mathrm{~nm}$ wavelength. The intensity fluctuation of the monochromator in this measurement was $2 \%$. With smaller slits $(10 \mu \mathrm{m})$, the minimum achieved instrumental FWHM is $10 \pm 1 \mathrm{pm}$. In addition to instrumental FWHM, the smaller slits reduce the throughput and therefore limit the number of detectable emission lines. Therefore, the wider $(100 \mu \mathrm{m})$ slits are used in this study. The instrumental FWHM is a function of the diffraction grating rotation angle and can be calculated from

$$
\lambda_{\mathrm{FWHM}}(\mathrm{nm})=\frac{d \lambda}{d x} \cdot w=\frac{10^{6} \cos \beta}{k n L} \cdot w,
$$

where $d \lambda / d x$ is the linear dispersion, $w$ is the exit slit width, $k$ is the diffraction order, $n$ is the groove density in grooves $/ \mathrm{mm}$, $L$ is the exit arm length in $\mathrm{mm}(994 \mathrm{~mm})$, and $\cos \beta$ is the angle

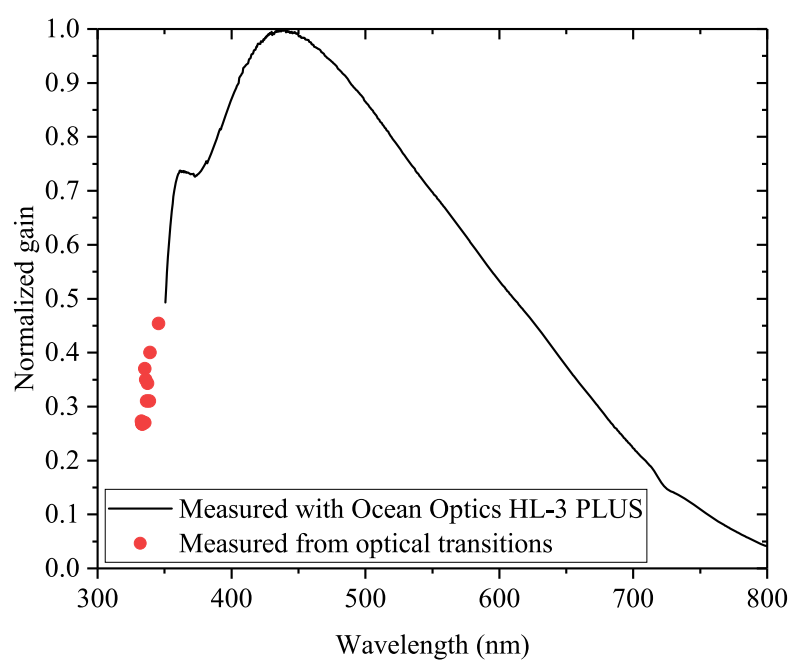

FIG. 6. The normalized spectral gain of the spectrometer. 
of diffraction. Based on the experimental result at $632.991 \mathrm{~nm}$ and Eq. (15), the instrumental broadening for the 1st order diffraction of the current setup was determined to range from $18 \mathrm{pm}$ (at $800 \mathrm{~nm}$ ) to $42 \mathrm{pm}$ (at $300 \mathrm{~nm}$ ). When as low instrumental broadening as possible is required, the second order diffraction can be used, which would reduce the broadening to half of the original value. However, in this case, the accessible wavelength would be decreased to $300-450 \mathrm{~nm}$ and the efficiency would be reduced by a factor of two.

\section{Data acquisition and spectrometer control}

The data acquisition system recording the signal from the photomultiplier tube consists of the Stanford Research Systems SR570 pre-amplifier and SR830 phase sensitive frequency lock-in amplifier. ${ }^{25}$ The photomultiplier raw signal is the current proportional to the incoming light signal. The preamplifier is a transimpedance type current to voltage amplifier with adjustable gain and frequency filtering. Typical gain used in the measurements is $1 \mu \mathrm{A} / \mathrm{V}$. Both low-pass and high-pass filters are used to clean the signal by band-passing the chopper frequency. The $-3 \mathrm{~dB}$ attenuations of the low- and high-pass filters are at $10 \mathrm{kHz}$ and $3 \mathrm{kHz}$, respectively, with a slope of $6 \mathrm{~dB} /$ octave. Using the pre-amplifier with filters allows increasing the dynamic reserve in comparison to direct connection from the photomultiplier to lock-in amplifier's current input.

The lock-in amplifier enables measuring small AC signals otherwise obscured by the noise from the photomultiplier tube. The use of the chopper and lock-in amplifier improves the SNR by a factor of $>1000$ in comparison to direct current measurement from the output of the PMT. In the case of the OES setup, the photomultiplier tube is operated in the current mode, i.e., the output is following the light input signal in time domain yielding a DC output signal when the light intensity is constant in time. Since an AC signal is required for the lock-in amplifier, the optical chopper is utilized to generate a square wave light signal input to the photomultiplier. The optical chopper provides also the reference signal. The time constant of the lock-in amplifier was $1 \mathrm{~s}$, and the slope of the used filter was $24 \mathrm{~dB} /$ octave. The data transfer between the lock-in amplifier and a computer go digitally through an IEEE488 GPIB bus to Agilent Technologies E5810A LAN/GPIB converter and along the LAN to the computer.

The rotation of the diffraction grating is realized with a $0.9^{\circ}$ /step NEMA 17 stepper motor and 1:1600 gearbox. The stepper motor is usually driven in the $1 / 16$ micro stepping mode. The maximum allowed grating rotation angle is $90^{\circ}$, and it is limited by optical gate sensors. A typical scanning speed resulting in appropriate signal-to-noise ratio is $660 \mathrm{~s} / \mathrm{nm}$. The stepper motor is controlled with Microstep Driver ST-7128 and Arduino UNO micro controller. The complete setup, including the data acquisition and control system, is controlled through LabVIEW graphical user interface.

\section{RESULTS AND DISCUSSION}

The applicability of the spectroscopic method for the study of low energy electron, as well as low charge state ion and
TABLE II. ECRIS tuning parameters used for the $\mathrm{Ne}^{7+}$ ion beam tune.

\begin{tabular}{lc}
\hline \hline Extraction voltage $(\mathrm{kV})$ & 10 \\
Gas feed rate $\left(\mathrm{cm}^{3} \mathrm{~h}^{-1}\right)$ & $0.18 \pm 0.01$ \\
Microwave power $(\mathrm{W})$ & 300 \\
Magnetic field inj. $(\mathrm{T})$ & 1.96 \\
Magnetic field ext. $(\mathrm{T})$ & 0.93 \\
Biased disc voltage $(\mathrm{V})$ & 164 \\
Biased disc current $(\mu \mathrm{A})$ & 256 \\
\hline \hline
\end{tabular}

neutral, populations in ECRIS plasma is demonstrated hereafter using the effect of the ion source extraction voltage as a representative example. The ion source was first optimized for $\mathrm{Ne}^{7+}$ corresponding to tune parameters presented in Table II. The measured ion beam currents of different charge states are presented in Table III. The optical emission spectrum was then recorded in the range of 330-800 nm with $10 \mathrm{kV}$ extraction voltage, which was then switched off without adjusting other source parameters before re-measuring the optical emission spectrum. The intensities of almost all emission lines of neon (neutral and $1+$ ion) were observed to increase significantly when the high voltage was switched off. Six emission lines (Table I) with different electron impact excitation cross sections, three originating from neutral neon and three from neon $1+$ ions, were chosen to closer examination. The intensity of transition 4, i.e., $\mathrm{Ne}^{1+}$ at $332.374 \mathrm{~nm}$, was measured 10 times to ensure that the fluctuation of the emission signal is small in the steady-state plasma condition. It was found that the standard deviation of the mean is $2 \%$ for the given transition. The intensity ratios of each six lines measured with and without the extraction voltage are presented in Fig. 7.

All emission line ratios presented in Fig. 7 are higher than 1.5 , i.e., the population rate of the corresponding excited states increases when the extraction voltage is switched off. The increased population rate is due to increased target density (neutral neon and $\mathrm{Ne}^{1+}$ ions), electron density, rate coefficient, or their combination [see Eq. (4)]. It is worth noting that the ratios of 1+ emission lines in Fig. 7 depend on the observed transition, which implies that the energy distribution of the cold electron population is affected by the extraction voltage. This is because the ratios would be independent of the specific transition if only the ion or electron densities would change without affecting the EEDF and the rate coefficient [see Eq. (13)].

TABLE III. Neon ion beam currents with gas feed rates of $0.18 \mathrm{~cm}^{3} \mathrm{~h}^{-1}$ and $0.11 \mathrm{~cm}^{3} \mathrm{~h}^{-1}$.

\begin{tabular}{lcc}
\hline \hline & \multicolumn{2}{c}{ Beam current $(\mu \mathrm{A})$} \\
\cline { 2 - 3 } Charge state & $0.18\left(\mathrm{~cm}^{3} \mathrm{~h}^{-1}\right)$ & $0.11\left(\mathrm{~cm}^{3} \mathrm{~h}^{-1}\right)$ \\
\hline $3+$ & 18 & 20 \\
$4+$ & 30 & 32 \\
$5+$ & 47 & 47 \\
$6+$ & 47 & 54 \\
$7+$ & 60 & 48 \\
$8+$ & 51 & 36 \\
$9+$ & 6 & 4 \\
\hline \hline
\end{tabular}




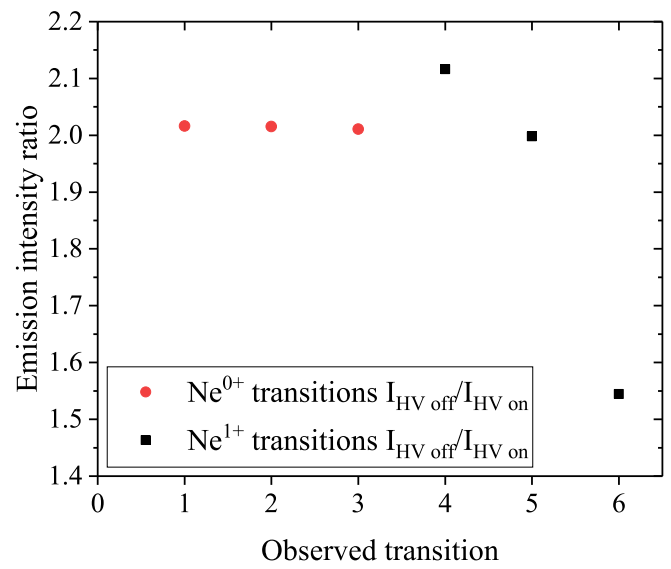

FIG. 7. The measured emission line intensity ratios $I_{\mathrm{HVoff}} / I_{\mathrm{HVon}}$ between extraction voltage off and on $(10 \mathrm{kV})$ for three emission lines of neutral neon and $\mathrm{Ne}^{1+}$

The effect of the extraction voltage on the electron temperature of the cold electrons can be estimated from the experimental results using the method described in Sec. II. The measured intensities presented in Fig. 7 are affected not only by direct excitation but also by cascade effects, i.e., radiative decays from higher electronic states populating the upper state of each detected transition. Unfortunately a large fraction of the cascade transitions are not in visible light range, and their effect cannot therefore be assessed directly. In the rate coefficient analysis yielding the electron temperature, we have taken these contributions into account, i.e., the estimated temperatures are based on direct electron impact excitation only. The electron temperature obtained (by using the Maxwell-Boltzmann velocity distribution) from the ratios of $\mathrm{Ne}^{1+}$ emission lines is $40 \pm 20 \mathrm{eV}$ and $20 \pm 10 \mathrm{eV}$ with the extraction voltage on and off, respectively. These results are shown in Fig. 8(a) with the corresponding error bars (obtained from the $\mathrm{min} / \mathrm{max}$ method). The vertical error bars refer to experimental uncertainty of the optical intensity measurement, while the horizontal error in electron temperature arises mainly from the uncertainties of the excitation cross sections $( \pm 10 \%)$ reported in Ref. 24 . The $10 \%$ corresponds to the worst case scenario with the majority of cross sections of excitation reactions having an uncertainty of less than 5\%. The asymmetric errors in electron temperature have been combined with a method described in Ref. 26. The horizontal error bar could not be defined reliably for all data points and is thus omitted in specific cases. The large relative uncertainty of the electron temperature estimate is due to small differential of the rate coefficient ratio [slope of the curves in Fig. 8(a)] in the relevant energy range. Two of the rate coefficient ratios decrease monotonically with increasing electron temperature and, hence, it can be concluded despite the relatively large uncertainties that the electron temperature (or average energy) of the cold electron population increases when the extraction voltage is switched on. The sensitivity of the results to the velocity distribution function was estimated by calculating the electron temperatures using the Druyvesteyn velocity distribution in addition to the Maxwell-Boltzmann distribution. The results from the comparison are presented in Fig. 8(b). The electron temperatures obtained with the Druyvesteyn distribution are $40 \pm 20 \mathrm{eV}$ and $30 \pm 10 \mathrm{eV}$ with the extraction voltage on and off, respectively. Thus, the results obtained for the two distributions are well within the error bars of each other. The presented method can be applied also to other assumed (or measured) distributions, i.e., the method can be generalized as long as the excitation cross sections are known, and the rate coefficients can be determined numerically. The electron temperatures estimated earlier are in the range of the values reported earlier in the literature. ${ }^{3,10}$ However, the presented method is more straightforward than modeling the entire population scheme (in Ref. 10), which in practice is applicable only for hydrogen and helium. Thus, the method can be considered to be more universal within the limits of its assumptions.

The increase in total population rate, seen as an increased optical emission intensity when the extraction voltage is switched off (Fig. 7), cannot be explained only by the change in the electron temperature. First of all, the calculated electron temperatures can be used to estimate the effect of the extraction voltage on the ratio between neutral and $1+$ ion densities $\left(n_{0} / n_{1+}\right)$ using Eq. (14). All combinations of the six observed transitions can be used to increase the a) Maxwell-Boltzmann distribution

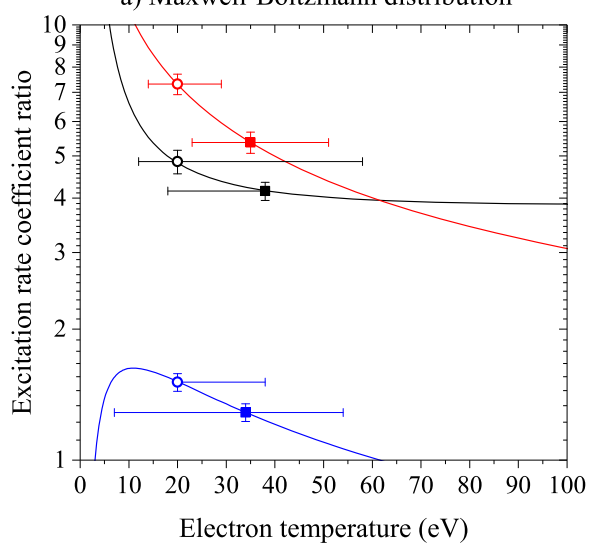

b) Druyvesteyn distribution

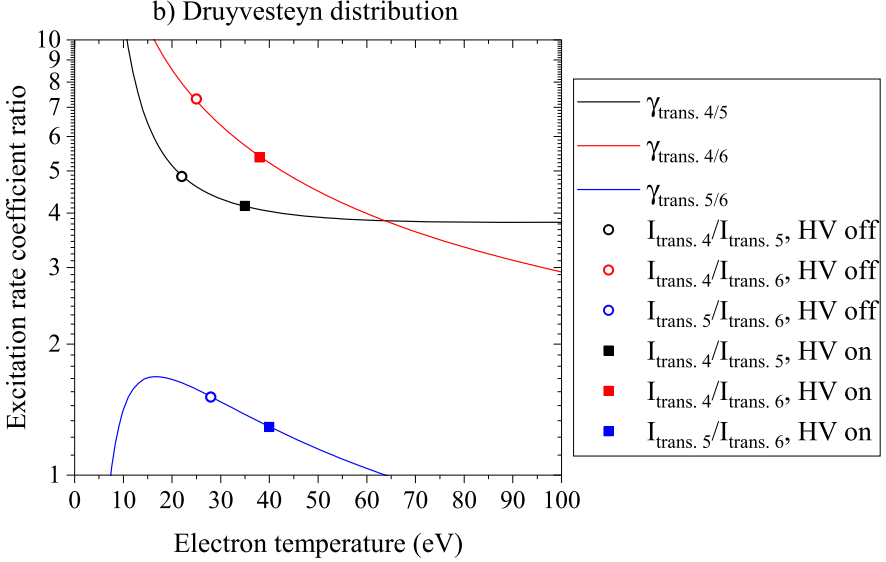

FIG. 8. The Maxwell-Boltzmann (a) and Druyvesteyn (b) excitation rate coefficient ratios of transitions 4, 5, and 6. The calculated electron temperatures with the extraction voltage on and off are indicated with empty dots. The error bars corresponding to the worst case scenario, i.e., $10 \%$ uncertainty of the excitation cross sections calculated with the ACE-code, are almost similar in both cases and are therefore only shown for the Maxwell-Boltzmann distribution. 
statistics. The ratio $n_{0} / n_{1+}$ is $(10 \pm 4) \%$ lower when the extraction voltage is switched off, which seemingly implies higher ionization degree despite the lower electron temperature. It can be deduced from the determined electron temperatures that when the extraction voltage is switched off, the neutral to $1+$ ionization rate coefficient decreases to $42 \%$ while the rate coefficient of $1+$ to $2+$ ionization decreases to $24 \%$. This suggests that switching the extraction voltage on favors ionization to charge states $\geq 2+$, which is then observed as an increase in the $n_{0} / n_{1+}$ ratio when the gas feed rate (source of first generation neutrals) is kept constant. This conclusion is also supported by the fact that the $1+$ emission intensity ratios are more sensitive to the effect of high voltage in comparison to neutral emission intensity ratios as depicted in Fig. 7. Second, the absolute emission intensity of all observed transitions was higher despite the lower cold electron temperature, when the extraction voltage was switched off. This implies either a higher target density or a higher electron density in this case. The reason for the observed trend was probed further by changing the gas feed rate.

The extraction voltage is believed to affect the particle dynamics of ECRIS plasmas due to the so-called ion pumping effect. This is because the extraction process can be considered as an ion sink affecting the loss rates and densities of charged particles. Since the frequency of inelastic collisions can be expected to depend on the density of the targets and, thus, is presumably affected by the ion pumping effect, it could be assumed that the change in the energy (temperature) of the electrons is associated with the ion pumping process. This was probed decreasing the neutral gas feed rate from $0.18 \pm 0.01 \mathrm{~cm}^{3} / \mathrm{h}$, used in the measurements described earlier, to $0.11 \pm 0.01 \mathrm{~cm}^{3} / \mathrm{h}$ without applying the extraction voltage so that the intensities of transitions 3 (neutral) and $4(1+$ ion) matched those obtained with higher gas feed rate and $10 \mathrm{kV}$ source potential. In other words, an attempt was made to see whether the effect of the extraction voltage could be matched simply by changing the gas feed rate. The resulting beam currents are listed in Table III. The ion beam intensities of $\mathrm{Ne}^{1+}$ and $\mathrm{Ne}^{2+}$ are not listed, as they cannot be $M / Q$-analyzed by the $90^{\circ}$ bending magnet of the JYFL $14 \mathrm{GHz}$ ECRIS with $10 \mathrm{kV}$ extraction potential. Lowering the extraction voltage would have resulted in different plasma equilibrium than probed by the optical emission studies performed at $10 \mathrm{kV}$ as well as enhanced transport losses of the high charge state ions in the low energy beamline. By decreasing the gas feed rate and switching the extraction voltage off simultaneously counteracted the overall increase of almost all of the emission line intensities, initially induced by switching off the extraction voltage. The measured emission line intensity ratios comparing the situations where the $\mathrm{HV}$ is off with $0.11 \mathrm{~cm}^{3} / \mathrm{h}$ feed rate and where the $\mathrm{HV}$ is on with $0.18 \mathrm{~cm}^{3} / \mathrm{h}$ feed rate are presented in Fig. 9. The results show that the reduction of the emission intensities of certain lines (e.g., transitions 3 and 4) induced by switching on the extraction voltage can be compensated by adjusting the neutral gas feed rate. However, the effect on the intensities of transitions 1 and 2 (neutrals) as well as 5 and $6(1+$ ions $)$ is not compensated simultaneously. This implies that variations in the electron or target (neutral/1+ion) densities are not sufficient to explain the change in electron

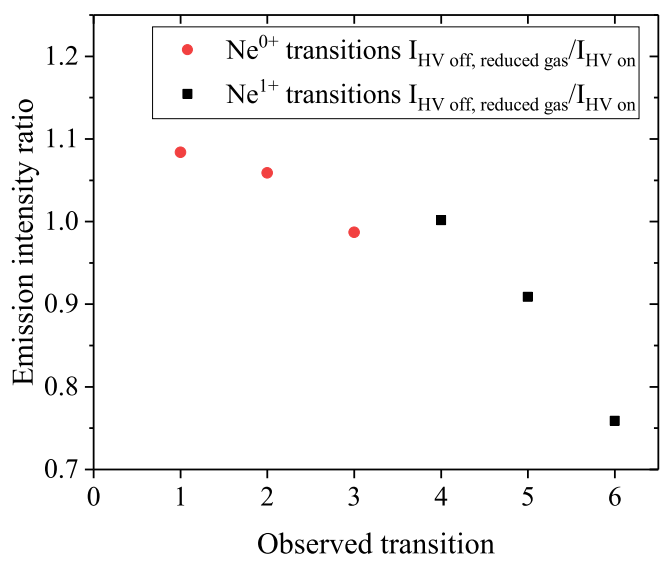

FIG. 9. The measured emission line intensity ratios between extraction voltage off and on $(10 \mathrm{kV})$ with a reduced gas feed rate $\left(0.11 \mathrm{vs} 0.18 \mathrm{~cm}^{3} \mathrm{~h}^{-1}\right)$ in the case of extraction voltage off.

temperature. The effect of the gas feed rate was probed further by adjusting the gas flow while keeping the extraction voltage constant at $10 \mathrm{kV}$. In this case, the ratios of the emission intensities are independent of the transition (Fig. 10), which leads to the conclusion that small adjustment of the gas feed rate does not affect the electron temperature substantially. Altogether it is concluded that the change of the particle densities due to ion pumping is not sufficient to explain the observed changes of the optical emission, but instead there are more complex mechanisms in action that alter also the electron temperature when the high voltage is switched on/off.

The major interest of most ECR ion source users is in the production of high charge state ions. Highly charged ions can also be studied with the diagnostics tool presented in this article, but the analysis is far more complicated in comparison to low charge states and neutrals. The detectable emission lines of high charge state noble gas ions in the visible light range originate from metastable states. This implies that the assumption of electron impact excitation from the ground state being the dominant channel populating these states is not valid anymore. Other excitation channels are (i) ionization of $q-1$ charge state ion resulting in electronic excitation of the charge state $q$, (ii) electron- $q+1$ ion recombination, (iii) charge exchange

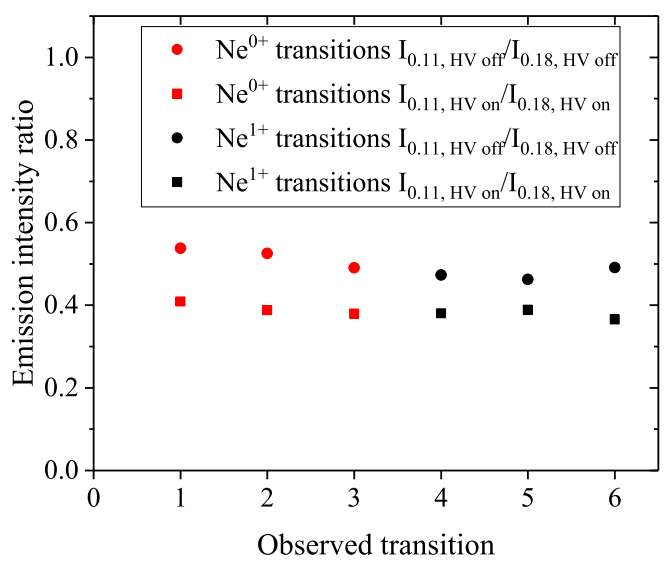

FIG. 10. The measured emission line intensity ratios between the gas feed rates of $0.11 \mathrm{~cm}^{3} \mathrm{~h}^{-1}$ and $0.18 \mathrm{~cm}^{3} \mathrm{~h}^{-1}\left(I_{0.11} / I_{0.18}\right)$ with the extraction voltage off (circles) and on (10 kV, squares). 


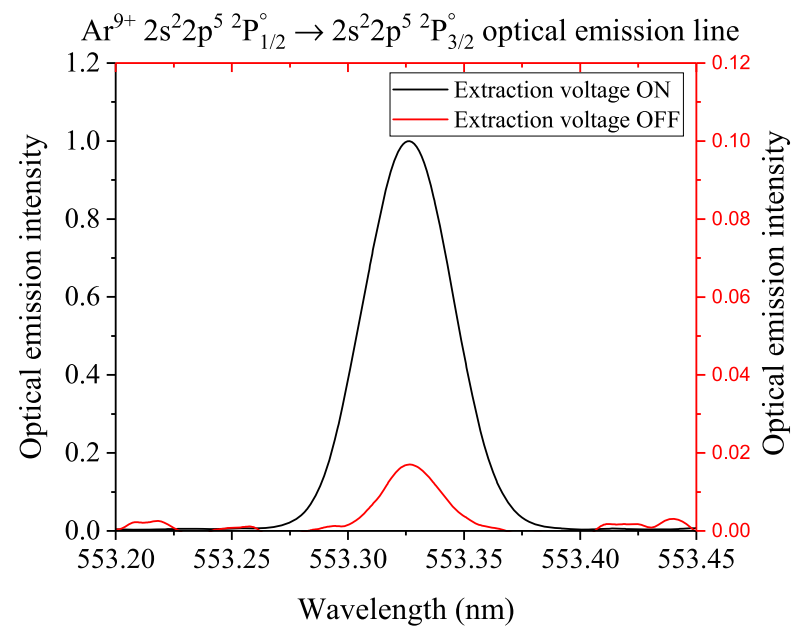

FIG. 11. The $\mathrm{Ar}^{9+} 2 \mathrm{~s}^{2} 2 \mathrm{p}^{5}{ }^{2} \mathrm{P}_{1 / 2}^{\circ} \rightarrow 2 \mathrm{~s}^{2} 2 \mathrm{p}^{5}{ }^{2} \mathrm{P}_{3 / 2}^{0}$ optical emission line at $553.3265 \mathrm{~nm}$ measured with the ion source HV on and off. All the other parameters including the magnetic field strength, microwave power $(500 \mathrm{~W})$, and neutral gas feed rate were kept constant between the two cases. Notice different vertical scales.

between $q+1$ ion and neutral/low charge state ion, and (iv) cascade effects from higher electronic states. As the contributions of each excitation mechanism can only be estimated, high charge state emission lines cannot be used for reliably determining the temperature of the relevant electron population. Nevertheless, the emission intensities of the electronic transitions of high charge state ions do reflect their densities. In other words, the effect of the source parameters such as high voltage on the high charge state ion density can be measured by comparing the emission intensities with $\mathrm{HV}$ on/off. However, the uncertainties related to deduced plasma parameters would be unacceptable in the case of high charge states. The situation is different in the case of low charge state ions as explained earlier. The intensities of optical emissions from highly charged neon ions are too small to be measured, but in the case of argon, the emission line originating from a metastable $2 s^{2} 2 \mathrm{p}^{5}{ }^{2} \mathrm{P}_{1 / 2^{\circ}}$ state of $\mathrm{Ar}^{9+}$ can be measured. In Fig. 11 of the manuscript, the effect of $\mathrm{HV}$ on the high charge state emission $\left(\mathrm{Ar}^{9+}\right.$ emission line corresponding magnetic dipole transitions between the metastable excited state and the ground state, $2 \mathrm{~s}^{2} 2 \mathrm{p}^{5}{ }^{2} \mathrm{P}_{1 / 2}^{\circ}$ $\rightarrow 2 s^{2} 2 p^{5}{ }^{2} \mathrm{P}_{3 / 2}^{\circ}$ ) is presented as an example in Fig. 11 of the manuscript. It is evident from the figure that switching off the high voltage reduces the emission intensity of $\mathrm{Ar}^{9+}$ ion by almost two orders of magnitude, which implies that the high charge state ion density is significantly affected by the ion source potential.

The main goal of this work is to demonstrate that a straightforward OES diagnostic can be used to estimate the temperature of cold electrons and study the densities and dynamics of low charge state ions in ECRIS plasmas. It is emphasized that the method can be applied for all plasmas (elements) with sufficiently intense optical transitions in the appropriate wavelength range. The effect of the extraction voltage on the plasma was used as an example, as it is a discrete change that was found to affect the aforementioned parameters. The results suggest that plasma diagnostics results of ECRIS plasmas obtained without the extraction voltage are not depicting the plasma conditions in normal ECRIS operation. It is therefore recommended that ECRIS plasma diagnostics should be performed with the extraction voltage switched on while diagnostics results obtained without the extraction voltage should be accompanied with larger error bars.

\section{ACKNOWLEDGMENTS}

The authors would like to thank J. Komppula, M. Liimatainen, and the JYFL electrical and mechanical workshop for their help in measurement technical issues. This project has received funding from European Union's Horizon 2020 research and innovation programme under Grant Agreement No. 654002 and the Academy of Finland under the Finnish Centre of Excellence Programme 2012-2017 (Nuclear and Accelerator Based Physics Research at JYFL).

${ }^{1}$ H. Koivisto, P. Heikkinen, V. Hänninen, A. Lassila, H. Leinonen, V. Nieminen, J. Pakarinen, K. Ranttila, J. Ärje, and E. Liukkonen, Nucl. Instrum. Methods Phys. Res., Sect. B 174, 379 (2001).

${ }^{2}$ C. Barué, M. Lamoureux, P. Briand, A. Girard, and G. Melin, J. Appl. Phys. 76, 2662 (1994).

${ }^{3}$ G. Douysset, H. Khodja, A. Girard, and J. P. Briand, Phys. Rev. E 61, 3015 (2000).

${ }^{4}$ G. Shirkov, Rev. Sci. Instrum. 71, 850 (2000).

${ }^{5}$ G. Melin, A. G. Drentje, A. Girard, and D. Hitz, J. Appl. Phys. 86, 4772 (1999).

${ }^{6}$ H. Tawara and T. Kato, At. Data Nucl. Data Tables 36, 167 (1987).

${ }^{7}$ E. Salzborn, IEEE Trans. Nucl. Sci. 23, 947 (1976)

${ }^{8}$ A. G. Drentje, A. Girard, D. Hitz, and G. Melin, Rev. Sci. Instrum. 71, 623 (2000).

${ }^{9}$ L. Kenéz, S. Biri, J. Karácsony, and A. Valek, Nucl. Instrum. Methods Phys. Res., Sect. B 187, 249 (2002).

${ }^{10}$ K. L. Junck, M. L. Brake, and W. D. Getty, Plasma Chem. Plasma Process. 11, 15 (1991).

${ }^{11}$ O. Tuske, L. Maunoury, J.-Y. Pacquet, C. Barué, M. Dubois, G. Gaubert, P. Jardin, N. Lecesne, P. Leherissier, F. Lemagnen, R. Leroy, M.-G. SaintLaurent, and A. C. C. Villari, Rev. Sci. Instrum. 75, 1529 (2004).

${ }^{12}$ H. Muto, Y. Ohshiro, S. Yamaka, S.-i. Watanabe, M. Oyaizu, S. Kubono, H. Yamaguchi, M. Kase, T. Hattori, and S. Shimoura, in 23rd International Conference on the Application of Accelerators in Research and IndustryCAARI 2014 [Phys. Procedia 66, 140 (2015)].

${ }^{13}$ N. K. Bibinov, V. F. Bratsev, D. B. Kokh, V. I. Ochkur, and K. Wiesemann, Plasma Sources Sci. Technol. 14, 109 (2005).

${ }^{14}$ K. E. Stiebing, L. Schächter, and S. Dobrescu, Rev. Sci. Instrum. 83, 02A331 (2012).

${ }^{15}$ O. Tarvainen, I. Izotov, D. Mansfeld, V. Skalyga, S. Golubev, T. Kalvas, H. Koivisto, J. Komppula, R. Kronholm, J. Laulainen, and V. Toivanen, Plasma Sources Sci. Technol. 23, 025020 (2014).

${ }^{16}$ U. Fantz, Plasma Sources Sci. Technol. 15, S137 (2006).

${ }^{17}$ A. Girard, D. Hitz, G. Melin, and K. Serebrennikov, Rev. Sci. Instrum. 75, 1381 (2004).

${ }^{18}$ V. Mironov, B. Sergey, A. Bondarchenko, A. Efremov, and V. Loginov, Phys. Rev. Spec. Top.-Accel. Beams 18, 123401 (2015).

${ }^{19}$ S. Mahmood, I. Orban, S. Ali, P. Glans, E. A. Bleda, Z. Altun, and R. Schuch, Astrophys. J. 771, 78 (2013).

${ }^{20} \mathrm{Z}$. Juhász, "Charge exchange processes that make comets radiate," Ph.D. thesis, University of Groningen, 2004.

${ }^{21}$ M. Moisan and J. Pelletier, Physics of Collisional Plasmas, Introduction to High-Frequency Discharges (Springer, The Netherlands, 2012).

${ }^{22} \mathrm{R}$. Cowan, The Theory of Atomic Structure and Spectra, Los Alamos Series in Basic and Applied Sciences (University of California Press, 1981).

${ }^{23}$ J. B. Mann, At. Data Nucl. Data Tables 29, 407 (1983).

${ }^{24}$ R. E. H. Clark, J. Abdallah, Jr., G. Csanak, J. B. Mann, and R. D. Cowan, Theoret. Atomic Phys. (1988).

${ }^{25}$ D. P. Blair and P. H. Sydenham, J. Phys. E: Sci. Instrum. 8, 621 (1975).

${ }^{26} \mathrm{R}$. Barlow, in Proceedings of Statistical Problems in Particle Physics, Astrophysics and Cosmology (PHYSTAT 05) Oxford, UK, September 12-15, 2005 (Imperial College Press, 2004), pp. 56-59, e-print arXiv: physics/0406120 [physics]. 